\title{
Synthesis and biological properties of 2-oxabicyclo[4.1.0]heptane nucleosides containing uracil, and thymine
}

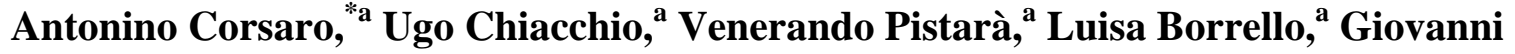 \\ Romeo, ${ }^{b}$ and Renato Dalpozzo ${ }^{c}$ \\ ${ }^{a}$ Dipartimento di Scienze Chimiche, Università di Catania, Viale Andrea Doria 6, Catania \\ 95125, Italy, ${ }^{b}$ Dipartimento Farmaco-Chimico, Università di Messina, Via SS. Annunziata, \\ Messina 98168, Italy, ${ }^{c}$ Dipartimento di Chimica, Universita della Calabria, Via P. Bucci, \\ Arcavacata di Rende (CS) 87036, Italy \\ E-mail: acorsaro@.unict.it
}

\section{Dedicated to Professor Giuseppe Bartoli on his 65th birthday}

\begin{abstract}
$\alpha$ - and $\beta$-2-Oxabicyclo[4.1.0]heptane nucleosides 8-13 containing uracil and thymine have been synthesized starting from L-threo-hex-4-enopyranosides 5 . The nucleosidation reaction performed with the $O$-acetyl derivative 7 affords only the $\beta$-anomer 8 while the nucleosidation of the $O$-methyl derivative 7 leads to the formation of $\alpha$ - and $\beta$-anomers. Antiviral, cytotoxicity and apoptotic activity have been investigated: no significant activity has been observed.
\end{abstract}

Keywords: Anti-HIV drugs, cyclopropanes, carbohydrates, nucleosides

\section{Introduction}

There has recently been a renewed interest in the synthesis of nucleosides with a six-membered carbohydrate moiety (Figure 1), due to their potential antiviral ${ }^{1-4}$ and antibiotic ${ }^{5}$ activities and as building blocks in nucleic acid synthesis. ${ }^{6}$ Antiviral properties have been found in 1,5anhydrohexitols 1 which exhibit a marked and selective activity against HSV-1, HSV-2, VZV, and CMV at relatively low concentrations. This activity is, probably, linked to a conformational flexibility comparable to that of natural nucleosides. ${ }^{2}$ 


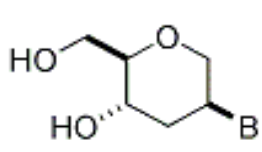

1

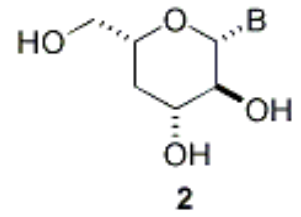

2
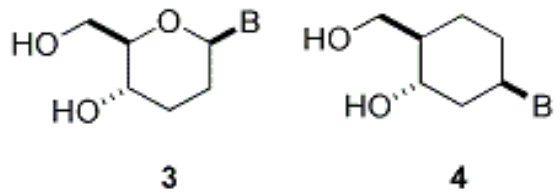

Figure 1. Structures of some anhydrohexitols $(\mathrm{B}=$ nucleobase $)$

The biological activity of nucleoside analogues is often modulated by the nature of the spacer (the furanose ring in natural compounds) which exerts a profound effect on the conformation and puckering of the molecule in natural nucleosides and which is able to control the outcome of the interactions between these compounds and the cellular kinases.

The correlation between the conformational preference required by the enzyme and the particular sugar conformation proves troublesome due to the flexibility of the furanose ring. Such flexibility is responsible for significant differences between the conformation in solid state and in solution. ${ }^{7}$ For this reason, conformationally restricted nucleoside analogues were synthesized with the aim to reduce their flexibility, so improving the interaction between substrates and enzymes. For instance, conformationally locked oxa- or carbocyclic nucleosides, built on a bicyclo[3.1.0] hexanes template, have demonstrated conformational preferences for a number of enzymes. ${ }^{8}$ Furthermore, the biological effects of modified nucleosides depend importantly from the relative distance and arrangement of the hydroxymethyl group and the base moiety.

In this context, we have devised the synthesis of a series of new nucleosides featuring the presence of a pyranose ring fused with a cyclopropane system, in order to construct a spacer unit which could control the conformational mobility while maintaining the base unit and the hydroxymethyl group in a disposition suitable to interact with the enzymes. In fact AM1 calculations performed on the synthesized 2-oxabicyclo[4.1.0]heptane nucleoside $\mathbf{1 3 b}$ and on the 1,5- $\beta$-pyranose nucleoside 2 (Figure 2) chosen as references, indicate that the introduction of the cyclopropane ring at $\mathrm{C} 4$ and $\mathrm{C} 5$, into $\mathbf{1 3 b}$ with respect to 2 , induces a puckering of the system which leads to a reduced conformational freedom and a lower distance between the base moiety and the hydroxymethyl group. 


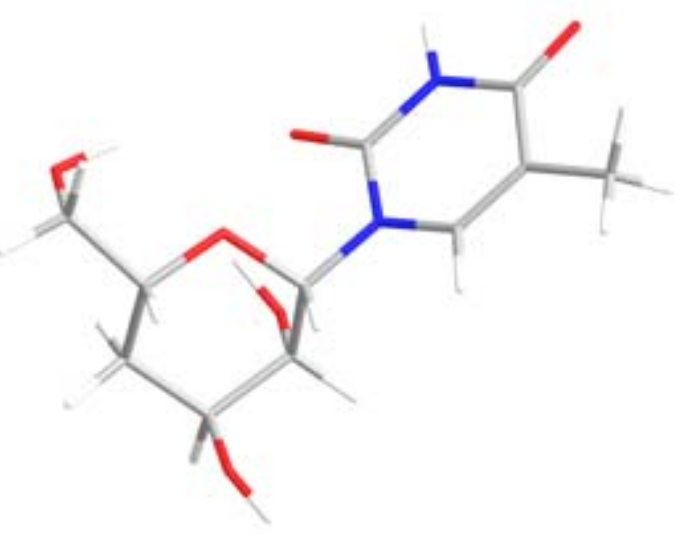

2

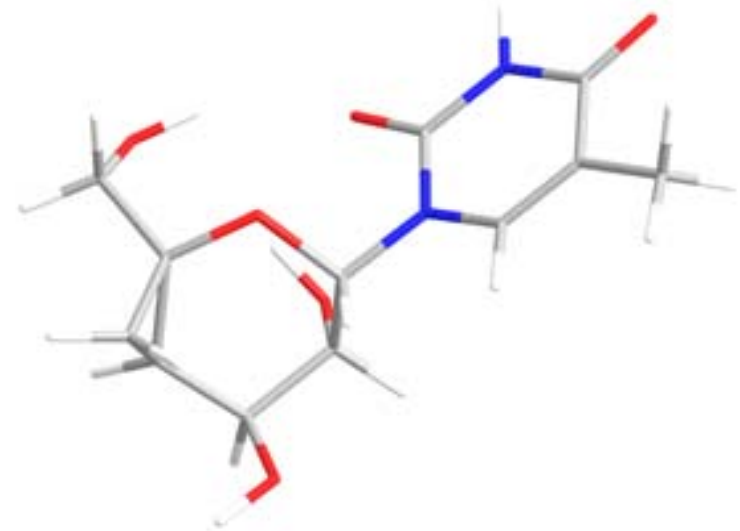

13b

Figure 2. AM1 optimized structures for compounds 2 and $\mathbf{1 3 b}$

According to these considerations, and following our interest in the chemical valorization of lactose, ${ }^{9}$ a natural disaccharide easily and cheaply available in large amounts as a waste product of the cheese industry, we report here the synthesis and the biological properties of the 2oxabicyclo[4.1.0]heptane nucleosides 8-13, not yet reported in the literature.

\section{Results and Discussion}

Recently we have described the cyclopropanation reactions ${ }^{10}$ of L-threo-hex-4-enopyranosides 5 , according to a Furukawa- modified ${ }^{11 \mathrm{a}}$ Simmons-Smith procedure, ${ }^{11 \mathrm{~b}}$ as a valuable tool to obtain 2-oxabicyclo[4.1.0]heptane derivatives 6, with a nearly quantitative yield (Scheme 1). These derivatives have then been utilized as the starting material for the preparation of nucleosides 813.

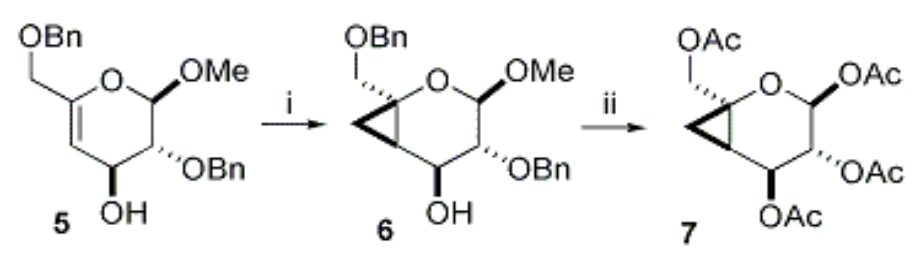

i) $\mathrm{CH}_{2} \mathrm{I}_{2}, \mathrm{Et}_{2} \mathrm{Zn}, \mathrm{Et}_{2} \mathrm{O}$; ii) a: (Ac) $)_{2} \mathrm{O}, \mathrm{Py}$; b: $(\mathrm{Ac})_{2} \mathrm{O}, \mathrm{H}_{2} \mathrm{SO}_{4}$.

\section{Scheme 1}

The cyclopropane derivative $\mathbf{6}$ was converted in the more reactive peracetylated compound $\mathbf{7}$ (Scheme 1) and used for the subsequent nucleosidation reaction with silylated uracil, under Vorbrüggen conditions, ${ }^{12}$ with TMSOTf in dry acetonitrile (Scheme 2). The reaction afforded 
exclusively the nucleoside 8 with good yield (80\% isolated) and high stereoselectivity. The stereochemistry of 8 was assessed on the basis of mono-and two- dimensional ${ }^{1} \mathrm{H}-\mathrm{NMR}$ experiments, and NOE effects. In particular, the diagnostic NOE enhancement observed for H-1 (4.4\%) when irradiating H-3 proton and vice versa, clearly indicates a trans relationship between the nucleobase and the substituent at C-6. Moreover, the H-1 proton resonates as a doublet (5.88 ppm) with a coupling constant $J_{1,2}=10.0 \mathrm{~Hz}$, typical of a trans ax $/$ ax disposition between $\mathrm{H}-1$ and H-2 protons.

The deprotection of $\mathbf{8}$ with $\mathrm{Et}_{3} \mathrm{~N}$ in methanol afforded the modified nucleoside $\mathbf{9}$ with a good yield (85\%) (Scheme 2$)$.

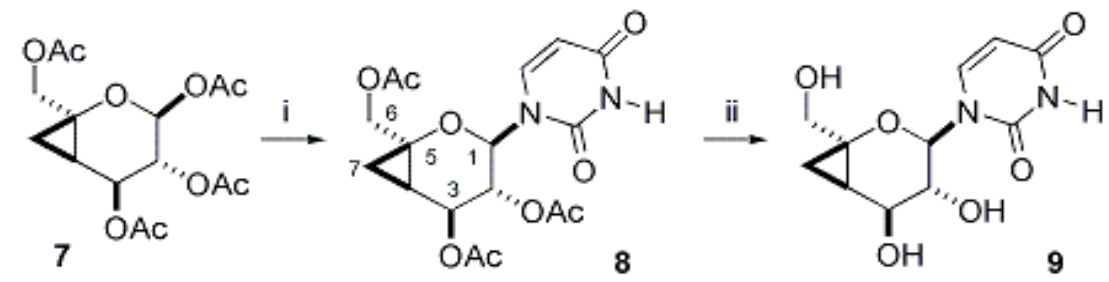

i) BSA, uracile, TMSOTf, MeCN, $\triangle 12 \mathrm{~h}$; ii) $\mathrm{Et}_{3} \mathrm{~N} / \mathrm{MeOH}$.

\section{Scheme 2}

The exclusive production of $\mathbf{8}$ is explainable in terms of the reversible formation of the $\sigma$ complex A which undergoes the attack of the persilylated base on the same side of the cyclopropane ring (Figure 3).

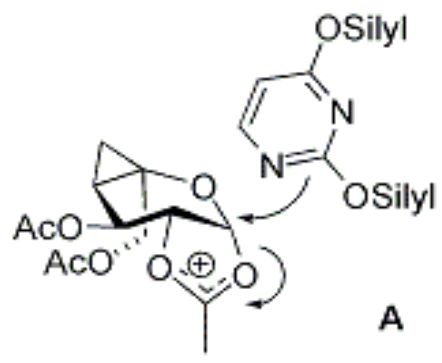

Figure 3. Proposed $\sigma$-complex between the persilylated uracil and the cyclopropanated sugar 7.

With the aim to obtain the modified nucleoside featuring a cis-disposition between the base and the hydroxymethyl group at C-6, and in order to compare the biological activities of cis- and trans- derivatives, we have performed the nucleosidation reaction of the more hindered cyclopropanated dibenzyl-methyl ether $\mathbf{6}^{10}$ with silylated uracil and thymine (Scheme 3). A mixture of the two $\alpha$ - and $\beta$ - anomers 10a,b and 11a,b in a $3: 1$ ratio (40\% total yield) was obtained: the pure compounds were isolated by flash and HPLC chromatography. 


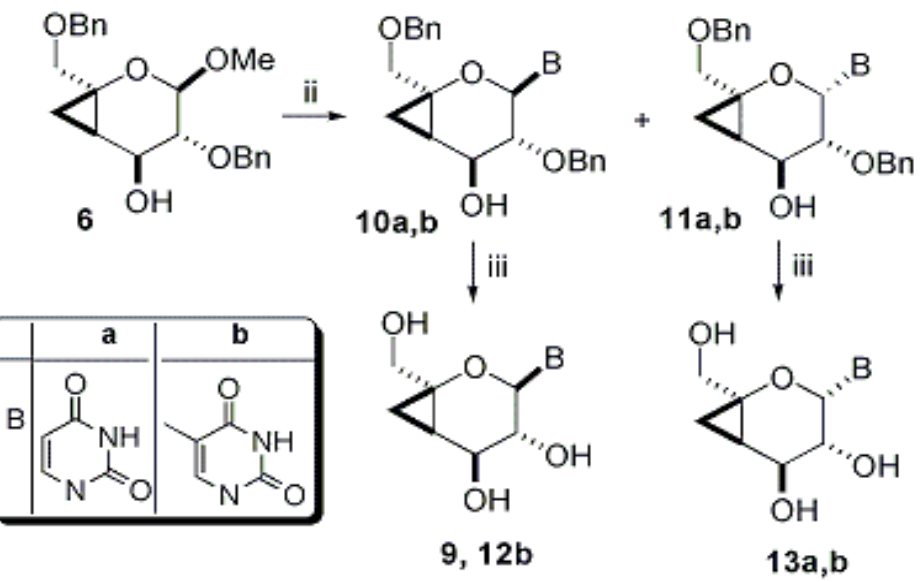

i) $\mathrm{CH}_{2} \mathrm{I}_{2}, \mathrm{Et}_{2} \mathrm{Zn}, \mathrm{Et}_{2} \mathrm{O}$; ii) BSA, nucleobase, TMSOTf, MeCN, $\Delta 12 \mathrm{~h}$; iii) $\mathrm{EtOH}, \mathrm{Pd} / \mathrm{C}, \mathrm{H}_{2}$.

\section{Scheme 3}

The stereochemistry was confirmed by mono-and two- dimensional ${ }^{1} \mathrm{H}$ - NMR experiments, and NOE measurements. In the case of the $\alpha$-nucleosides $(\mathbf{1 0 a}, \mathbf{b})$, the anomeric proton appears as a doublet (5.66 ppm) with a coupling constant $J_{1,2}=9.5 \mathrm{~Hz}$, typical of a trans $_{\mathrm{ax}}$ / ax disposition between H-1 and H-2 protons; on the contrary in $\beta$-nucleosides (11a,b), $\mathrm{H}-1$ appears as a doublet $(5.93 \mathrm{ppm})$ with a small coupling constant $J_{\mathrm{ax} / \text { eq }}(2.5 \mathrm{~Hz})$. Moreover, for 10a,b diagnostic NOE enhancements were observed for H-1 when irradiating H-3 (2.5\%). No NOE effect was observed in $\beta$-anomers 11a,b between $\mathrm{H}-1$ and $\mathrm{H}-3$ protons (Figure 4 ).
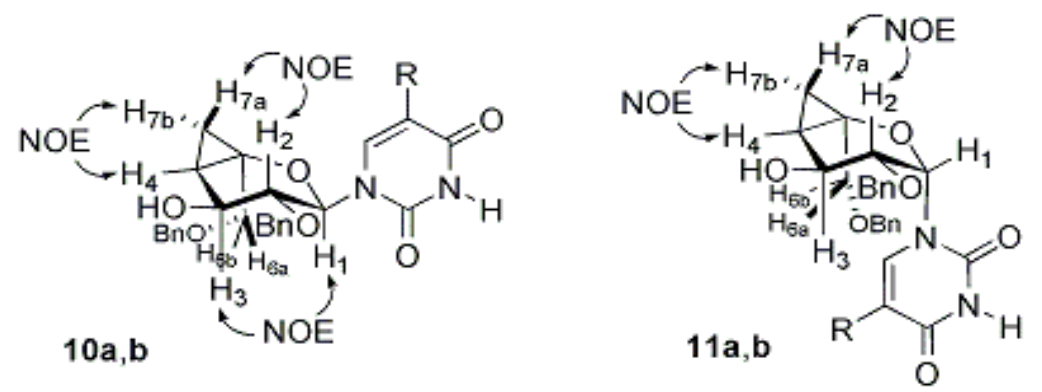

10,11: $\mathbf{a}=\mathrm{H}, \mathbf{b}=\mathrm{CH}_{3}$

Figure 4. NOE enhancements for 10a,b and 11a,b.

The fully deprotected 12a,b and 13a,b nucleosides were then obtained in a nearly quantitative yields by catalytic debenzylation reaction (Scheme 3 ).

\section{Biological assays}


The synthesized compounds were tested for their antiviral activity against HSV-1, HSV-2 in Vero (African Green Monkey) cells, HTLV-1 in human cells (lymphomonocytes of peripheral blood) and for their toxicity in vitro. None of these derivatives reached the inhibitory concentration 50 at the highest concentrations tested (e.g. 320 1M), indicating a lack of significant antiviral activity.

The apoptotic activity was also tested in Molt-3 cells. Only 13b showed a moderate activity (32\%) at the concentration $500 \mu \mathrm{M}$ after $48 \mathrm{~h}$. No significant toxicity, when evaluated using the classical trypan blue test, was detected until 3 days of treatment in lymphoid Molt-3 cell line assayed.

\section{Conclusions}

The synthesis of $\alpha$ - and $\beta$-2-oxabicyclo[4.1.0]heptane nucleosides 8-13 containing the uracil and thymine was achieved starting from L-threo-hex-4-enopyranosides 5 . The nucleosidation of $O$ acetyl derivative 7 affords only $\beta$-anomer 8 while the nucleosidation of $O$-methyl derivative 7 leads to the formation of $\alpha$ - and $\beta$-anomers.

Only 13b exhibits a moderate apoptotic activity (28\%) at the concentration $500 \mu \mathrm{M}$ after $48 \mathrm{~h}$.

\section{Experimental Section}

General Procedures. Melting points were determined with a Kofler apparatus and are uncorrected. Elemental analyses were performed with a Perkin-Elmer elemental analyzer. NMR spectra were recorded on a Varian instrument at 200 or $500 \mathrm{MHz}\left({ }^{1} \mathrm{H}\right)$ and at 50 or $125 \mathrm{MHz}$ $\left({ }^{13} \mathrm{C}\right)$ using $\mathrm{CDCl}_{3}, \mathrm{CD}_{3} \mathrm{OD}$ or $\mathrm{CD}_{3} \mathrm{CN}$ as solvent; chemical shifts are given in ppm from TMS as internal standard. Thin-layer chromatographic separations were performed on Merck silica gel $60-\mathrm{F}_{254}$ precoated aluminium plates. Preparative separations were by column- and flash chromatography using Merck silica gel 0.063-0.200 mm and 0.035-0.070 mm, respectively, with chloroform-methanol mixtures as eluents. HPLC purifications were made with a preparative

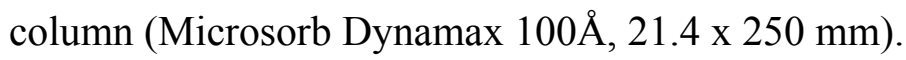

Starting materials. Thymine, uracil and all the reagents were purchased from Aldrich Co. All solvents were dried according to literature methods.

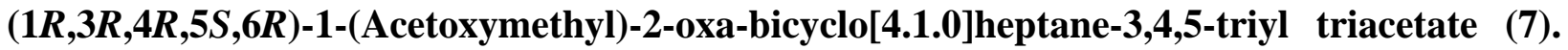
To a solution of compound $\mathbf{6}^{10}(750 \mathrm{mg}, 2 \mathrm{mmol})$ in pyridine $(9.5 \mathrm{ml})$, was added acetic anhydride (1,9 $\mathrm{ml}, 19.8 \mathrm{mmol})$ under stirring at room temperature. After the disappearance of the starting product $(3 \mathrm{~h})$, the reaction mixture was evaporated under reduced pressure and coevaporated with toluene; then was dissolved in acetic acid $(9.5 \mathrm{ml})$, acetic anhydride $(1,9 \mathrm{ml}$, 
$20.8 \mathrm{mmol})$ was added and, dropwise at $0{ }^{\circ} \mathrm{C}$, sulfuric acid $(0,039 \mathrm{ml})$. The reaction mixture was stirred at room temperature for $16 \mathrm{~h}$, then diluted with ice and extracted $(2 \mathrm{x} 10 \mathrm{ml})$ with $\mathrm{CH}_{2} \mathrm{Cl}_{2}$. The organic phases were washed with a solution of $\mathrm{NaHCO}_{3}$ and dried over $\mathrm{Na}_{2} \mathrm{SO}_{4}$, filtered and evaporated under vacuum. The residue, purified by flash chromatography, affords the exclusive $\alpha$-anomer 7 (75\% yield) as a yellow syrup: ${ }^{1} \mathrm{H} \mathrm{NMR}\left(\mathrm{CDCl}_{3}, 500 \mathrm{MHz}\right) \delta 0.98(\mathrm{dd}, 1 \mathrm{H}, J=6.8$, $10.0 \mathrm{~Hz}, \mathrm{H}-7 \mathrm{a}$ ), 1.06 (dd, 1H, $J=6.8,7.0 \mathrm{~Hz}, \mathrm{H}-7 \mathrm{~b}$ ), 1.93 (ddd, $1 \mathrm{H}, J=6.8,7.0,10.0 \mathrm{~Hz}, \mathrm{H}-4$ ), 2.04, 2.09, 2.16, and 2.17 (s, each 3H, $4 \times \mathrm{CH}_{3}$ ), 4.05-4.13 (AB system, $2 \mathrm{H}, J_{\mathrm{AB}}=12.3 \mathrm{~Hz}, \mathrm{H}-6 \mathrm{a}$, $\mathrm{H}-6 \mathrm{~b}), 4.84$ (dd, $1 \mathrm{H}, J=2.8,9.0 \mathrm{~Hz}, \mathrm{H}-2), 5.48$ (dd, 1H, $J=7.0,9.0 \mathrm{~Hz}, \mathrm{H}-3), 6.03$ (d, $1 \mathrm{H}, J=$ $2.8 \mathrm{~Hz}, \mathrm{H}-1) ;{ }^{13} \mathrm{C} \mathrm{NMR}\left(\mathrm{CDCl}_{3}, 50 \mathrm{MHz}\right) \delta 15.68$ (C-7), 20.32 (C-4), 20.64, 20.86, 21.06, and $21.12\left(4 \mathrm{x} \mathrm{CH}_{3}\right), 60.22$ (C-5), 66.83 (C-3), 68.04 (C-6), 68.92 (C-2), 89.67 (C-1), 169.35, 170.03, 170.68, $170.87(\mathrm{C}=\mathrm{O})$; Anal. Calc. for $\mathrm{C}_{15} \mathrm{H}_{20} \mathrm{O}_{9}$ : C, 52.32; H, 5.85\%. Found: $\mathrm{C}, 52.37$; H, 5.82.

General procedure for the reaction between silylated bases (uracil and thymine) with cyclopropanated sugar 6 and 7. A suspension of base $(0.62 \mathrm{mmol})$ in dry acetonitrile $(3 \mathrm{~mL})$ was treated with $2.48 \mathrm{mmol}$ of bis(trimethylsilyl)acetamide (BSA) and refluxed for 15 min under stirring. To the clear solution obtained was added at room temperature a solution of the cyclopropanated sugar $(0.52 \mathrm{mmol})$ in dry acetonitrile $(3 \mathrm{~mL}), 0.78 \mathrm{mmol}$ of trimethylsilyl triflate (TMSOTf) dropwise, and the reaction mixture was refluxed for $12 \mathrm{~h}$. After being cooled at $0{ }^{\circ} \mathrm{C}$, the solution was neutralized by careful addition of aqueous $5 \%$ sodium bicarbonate and then concentrated under vacuum. After addition of dichloromethane $(8 \mathrm{~mL})$ the organic phase was separated, washed with water $(2 \times 10 \mathrm{~mL})$, dried over sodium sulfate, filtered, and evaporated to dryness. The residue was purified by flash chromatography to give the cyclopropanated nucleoside (9), and for the anomeric mixture of compounds 10a,b and 11a,b, by HPLC (2$\operatorname{propanol} / n$-hexane).

(1R,3R,4R,5S,6R)-1-(Acetoxymethyl)-3-[2,4-dioxo-3,4-dihydropyrimidin-1(2H)-yl]-2-oxabicyclo[4.1.0]heptane-4,5-diyl diacetate (8). Amorphous solid, yield $80 \%$, $[\alpha]_{\mathrm{D}}^{25}+52.63^{\circ}(\mathrm{c} 0.1$, $\left.\mathrm{CHCl}_{3}\right) .{ }^{1} \mathrm{H} \mathrm{NMR}\left(\mathrm{CDCl}_{3}, 500 \mathrm{MHz}\right) \delta 1.03(\mathrm{dd}, 1 \mathrm{H}, J=7.0,10.5 \mathrm{~Hz}, \mathrm{H}-7 \mathrm{a}), 1.16(\mathrm{dd}, 1 \mathrm{H}, J=$ 7.0, 7.5 Hz, H-7b), 1.93 (ddd, 1H, J=7.5, 8.0, 10.5 Hz, H-4), 2.04, 2.09, and 2.15 (s, each 3H, 3 x $\mathrm{CH}_{3}$ ), 3.78-4.51 (AB system, $2 \mathrm{H}, J_{\mathrm{AB}}=12.3 \mathrm{~Hz}, \mathrm{H}-6 \mathrm{a}, \mathrm{H}-6 \mathrm{~b}$ ), 4.95 (dd, $1 \mathrm{H}, J=8.0,10.0 \mathrm{~Hz}$, H-2), 5.56 (dd, 1H, $J=7.5,8.0 \mathrm{~Hz}, \mathrm{H}-3), 5.72$ (dd, 1H, $J=8.3,2.1 \mathrm{~Hz}, \mathrm{H}-5$ ') 5.89 (d, 1H, $J=$ $10.0 \mathrm{~Hz}, \mathrm{H}-1), 7.22(\mathrm{~d}, 1 \mathrm{H}, J=8.3 \mathrm{~Hz}, \mathrm{H}-6)$ ') $8.54(\mathrm{bs}, 1 \mathrm{H}, \mathrm{NH}) ;{ }^{13} \mathrm{C} \mathrm{NMR}\left(\mathrm{CDCl}_{3}, 50 \mathrm{MHz}\right) \delta$ 14.18 (C-7), 16.09 (C-4), 20.77, 20.89, and 21.16 (3 x CH 3$), 60.39$ (C-5), 67.33 (C-6), 69.38 (C3), 71.47 (C-2), 79.46 (C-1), 103.07 (C-5'), 139.31 (C-6'), 150.10 (C-2'), 162.24 (C-4'), 169.78, 170.86, and $171.05(\mathrm{C}=\mathrm{O})$; Anal. Calc. for $\mathrm{C}_{17} \mathrm{H}_{20} \mathrm{~N}_{2} \mathrm{O}_{9}$ : C, 51.52; H, 5.09\%. Found: C, 51.59; H, 5.11 .

1-\{(1R,3R,4R,5S,6R)-4-(Benzyloxy)-1-(benzyloxymethyl)-5-hydroxy-2-oxa-bicyclo[4.1.0]heptan-3-yl\}pyrimidine-2,4(1H,3H)-dione (10a). Amorphous solid, yield 33\%, $[\alpha]_{\mathrm{D}}^{25}+123.90^{\circ}$ (c $\left.0.1, \mathrm{CHCl}_{3}\right) .{ }^{1} \mathrm{H} \mathrm{NMR}\left(\mathrm{CDCl}_{3}, 500 \mathrm{MHz}\right) \delta 0.95(\mathrm{dd}, 1 \mathrm{H}, J=6.0,10.5 \mathrm{~Hz}, \mathrm{H}-7 \mathrm{a}), 1.03$ (dd, $1 \mathrm{H}, J=6.0,7.0 \mathrm{~Hz}, \mathrm{H}-7 \mathrm{~b}), 1.59$ (ddd, 1H, $J=6.0,7.0,10.5 \mathrm{~Hz}, \mathrm{H}-4), 3.36$ (dd, 1H, $J=7.0,9.5$ 
Hz, H-2), 3.42-3.69 (AB system, 2H, $\left.J_{\mathrm{AB}}=10.5 \mathrm{~Hz}, \mathrm{H}-6 \mathrm{a}, \mathrm{H}-6 \mathrm{~b}\right), 4.38$ (dd, 1H, J = 6.5, 7.0 Hz, $\mathrm{H}-3)$, 4.52-4.81 (2 x AB system, each $\left.2 \mathrm{H}, J_{\mathrm{AB}}=12.0 \mathrm{~Hz}, \mathrm{OCH}_{2} \mathrm{Ph}\right), 5.43(\mathrm{~d}, 1 \mathrm{H}, J=8.0 \mathrm{~Hz}, \mathrm{H}-$ 5'), 5.72 (d, 1H, $J=9.5 \mathrm{~Hz}, \mathrm{H}-1), 6.89$ (d, 1H, $J=8.0 \mathrm{~Hz}, \mathrm{H}-6$ '), 7.13-7.38 (m, 10H, aromatic $\mathrm{H}), 8.19$ (bs, $1 \mathrm{H}, \mathrm{NH}) ;{ }^{13} \mathrm{C}$ NMR $\left(\mathrm{CDCl}_{3}, 50 \mathrm{MHz}\right) \delta 15.25$ (C-7), 19.97 (C-4), 63.62 (C-5), 70.33 (C-6), 73.10 and $73.29\left(\mathrm{CH}_{2}\right), 77.73$ (C-3), 78.23 (C-2), 80.79 (C-1), 102.34 (C-5'), 127.67, 127.88, 128.03, 128.20, 128.39 and 128.51 (aromatic $\mathrm{CH}$ ), 137.18 and 137.73 (aromatic C), 139.20 (C-6'), 150.12 and $162.48(\mathrm{C}=\mathrm{O})$; Anal. Calc. for $\mathrm{C}_{25} \mathrm{H}_{26} \mathrm{~N}_{2} \mathrm{O}_{6}$ : C, 66.65; H, 5.82\%. Found: C, 66.71; H, 5.85.

1-\{(1R,3S,4R,5S,6R)-4-(Benzyloxy)-1-(benzyloxymethyl)-5-hydroxy-2-oxa-bicyclo[4.1.0]heptan-3-yl\}pyrimidine-2,4(1H,3H)-dione (11a). Amorphous solid, yield $15 \%,[\alpha]_{\mathrm{D}}^{25}-83.55^{\circ}$ (c $\left.0.5, \mathrm{CHCl}_{3}\right) .{ }^{1} \mathrm{H} \mathrm{NMR}\left(\mathrm{CDCl}_{3}, 500 \mathrm{MHz}\right) \delta 0.89(\mathrm{dd}, 1 \mathrm{H}, J=6.5,10.5 \mathrm{~Hz}, \mathrm{H}-7 \mathrm{a}), 1.05(\mathrm{dd}, 1 \mathrm{H}$, $J=6.5,7.0 \mathrm{~Hz}, \mathrm{H}-7 \mathrm{~b}), 1.61(\mathrm{ddd}, 1 \mathrm{H}, J=6.5,7.0,10.5 \mathrm{~Hz}, \mathrm{H}-4), 3.31$ (dd, $1 \mathrm{H}, J=2.5,7.0 \mathrm{~Hz}$, H-2), 3.38-3.55 (AB system, 2H, $\left.J_{\mathrm{AB}}=11.5 \mathrm{~Hz}, \mathrm{H}-6 \mathrm{a}, \mathrm{H}-6 \mathrm{~b}\right), 4.49$ (dd, 1H, J=7.0, $7.5 \mathrm{~Hz}, \mathrm{H}-$ 3), 4.52-4.81 (2 x AB system, each $\left.2 \mathrm{H}, J_{\mathrm{AB}}=12.0 \mathrm{~Hz}, \mathrm{OCH}_{2} \mathrm{Ph}\right), 5.66$ (d, $1 \mathrm{H}, J=8.0 \mathrm{~Hz}, \mathrm{H}-5$ '), $5.85(\mathrm{~d}, 1 \mathrm{H}, J=2.5 \mathrm{~Hz}, \mathrm{H}-1), 7.09(\mathrm{~d}, 1 \mathrm{H}, J=8.0 \mathrm{~Hz}, \mathrm{H}-6$ '), 7.21-7.38 (m, 10H, aromatic H), 8.25 (bs, 1H, NH); ${ }^{13} \mathrm{C} \mathrm{NMR}\left(\mathrm{CDCl}_{3}, 50 \mathrm{MHz}\right) \delta 16.25$ (C-7), 21.27 (C-4), $62.62(\mathrm{C}-5), 67.73$ (C-3), 70.29 (C-6), 73.25 and $73.43\left(\mathrm{CH}_{2}\right), 79.32$ (C-2), 83.56 (C-1), 103.34 (C-5'), 126.88, 127.08, 127.93, 128.02, 128.30 and 128.41 (aromatic $\mathrm{CH}$ ), 137.68 and 137.93 (aromatic $\mathrm{C}$ ), 138.27 (C-6'), 160.42 and $161.39(\mathrm{C}=\mathrm{O})$; Anal. Calc. for $\mathrm{C}_{25} \mathrm{H}_{26} \mathrm{~N}_{2} \mathrm{O}_{6}$ : C, 66.65; H, 5.82\%. Found: C, 66.71; H, 5.85 .

1-\{(1R,3R,4R,5S,6R)-4-(Benzyloxy)-1-(benzyloxymethyl)-5-hydroxy-2-oxa-bicyclo[4.1.0]heptan-3-yl\}-5-methylpyrimidine-2,4(1H,3H)-dione (10b). Amorphous solid, yield 30\%, $[\alpha]_{\mathrm{D}}^{25}$ $+26.82^{\circ}\left(\mathrm{c} 0.7, \mathrm{CHCl}_{3}\right) .{ }^{1} \mathrm{H} \mathrm{NMR}\left(\mathrm{CDCl}_{3}, 500 \mathrm{MHz}\right) \delta 0.90(\mathrm{dd}, 1 \mathrm{H}, J=6.5,10.0 \mathrm{~Hz}, \mathrm{H}-7 \mathrm{a})$, $1.01(\mathrm{dd}, 1 \mathrm{H}, J=6.5,7.0 \mathrm{~Hz}, \mathrm{H}-7 \mathrm{~b}), 1.60(\mathrm{ddd}, 1 \mathrm{H}, J=6.5,7.0,10.0 \mathrm{~Hz}, \mathrm{H}-4), 1.77$ (d, $J=1.0$ $\mathrm{Hz}, \mathrm{CH}_{3}$ ), 3.28 (dd, $1 \mathrm{H}, J=7.5,9.5 \mathrm{~Hz}, \mathrm{H}-2$ ), 3.34-3.65 (AB system, $2 \mathrm{H}, J_{\mathrm{AB}}=10.5 \mathrm{~Hz}, \mathrm{H}-6 \mathrm{a}$, $\mathrm{H}-6 \mathrm{~b}), 4.55(\mathrm{dd}, 1 \mathrm{H}, J=7.5,8.0 \mathrm{~Hz}, \mathrm{H}-3), 4.52-4.58$ (AB system, 2H, $J_{\mathrm{AB}}=12.0 \mathrm{~Hz}, \mathrm{C} 6-$ $\mathrm{OCH}_{2} \mathrm{Ph}$ ), 4,56-4,71 (AB system, $\left.2 \mathrm{H}, J_{\mathrm{AB}}=12.0 \mathrm{~Hz}, \mathrm{C} 2-\mathrm{OCH}_{2} \mathrm{Ph}\right), 5.66(\mathrm{~d}, 1 \mathrm{H}, J=9.5 \mathrm{~Hz}, \mathrm{H}-$ 1), 6.75 (d, $1 \mathrm{H}, J=1.0 \mathrm{~Hz}, \mathrm{H}-6$ '), $7.17-7.33$ (m, 10H, aromatic H), 8.64 (bs, $1 \mathrm{H}, \mathrm{NH}) ;{ }^{13} \mathrm{C}$ NMR $\left(\mathrm{CDCl}_{3}, 50 \mathrm{MHz}\right) \delta 12.50\left(\mathrm{CH}_{3}\right), 15.15(\mathrm{C}-7), 23.14(\mathrm{C}-4), 62.80$ (C-5), 71.67 (C-3), 73.13, 73.18 and $73.55\left(\mathrm{CH}_{2}\right), 79.08(\mathrm{C}-2), 80.95(\mathrm{C}-1), 110.77\left(\mathrm{C}-5^{\prime}\right), 127.60,127.67,128.10,128.35$ and 128.45 (aromatic CH), 135.27 (C-6') 137.25, 137.82 (aromatic C), 150.41 (C-2'), 163.35 (C4'); Anal. Calc. for $\mathrm{C}_{26} \mathrm{H}_{28} \mathrm{~N}_{2} \mathrm{O}_{6}$ : C, 67.23; H, 6.08\%. Found: C, 67.33; H, 6.12.

1-\{(1R,3S,4R,5S,6R)-4-(Benzyloxy)-1-(benzyloxymethyl)-5-hydroxy-2-oxa-bicyclo[4.1.0]heptan-3-yl\}-5-methylpyrimidine-2,4(1H,3H)-dione (11b). Amorphous solid, yield 10\%, $[\alpha]_{\mathrm{D}}^{25}$ $-59.09^{\circ}\left(\mathrm{c} 0.2, \mathrm{CHCl}_{3}\right) .{ }^{1} \mathrm{H} \mathrm{NMR}\left(\mathrm{CDCl}_{3}, 500 \mathrm{MHz}\right) \delta 0.77(\mathrm{dd}, 1 \mathrm{H}, J=6.5,10.0 \mathrm{~Hz}, \mathrm{H}-7 \mathrm{a})$, 1.23 (dd, $1 \mathrm{H}, J=6.5,7.0 \mathrm{~Hz}, \mathrm{H}-7 \mathrm{~b}), 1.53$ (ddd, $1 \mathrm{H}, J=6.5,7.0,10.0 \mathrm{~Hz}, \mathrm{H}-4), 1.73$ (d, $J=1.5$ $\left.\mathrm{Hz}, \mathrm{CH}_{3}\right), 2.45$ (bs, $\left.1 \mathrm{H}, \mathrm{OH}\right), 3.41(\mathrm{dd}, 1 \mathrm{H}, J=2.5,4.5 \mathrm{~Hz}, \mathrm{H}-2), 3.50-3.81$ (AB system, 2H, $J_{\mathrm{AB}}$ $=11.0 \mathrm{~Hz}, \mathrm{H}-6 \mathrm{a}, \mathrm{H}-6 \mathrm{~b}), 4.46-4.60\left(\mathrm{AB}\right.$ system, $\left.2 \mathrm{H}, J_{\mathrm{AB}}=11.5 \mathrm{~Hz}, \mathrm{C} 3-\mathrm{OCH}{ }_{2} \mathrm{Ph}\right), 4.51(\mathrm{dd}, 1 \mathrm{H}, J$ $=4.5,7.0 \mathrm{~Hz}, \mathrm{H}-3), 4.56-4.63\left(\mathrm{AB}\right.$ system, $\left.2 \mathrm{H}, J_{\mathrm{AB}}=12.0 \mathrm{~Hz}, \mathrm{C} 6-\mathrm{OCH}{ }_{2} \mathrm{Ph}\right), 5.93(\mathrm{~d}, 1 \mathrm{H}, J=2.5$ $\mathrm{Hz}, \mathrm{H}-1), 7.19-7.63$ (m, 10H, aromatic H), 7.63 (d, 1H, $J=1.5 \mathrm{~Hz}, \mathrm{H}-6$ '), 8.46 (bs, $1 \mathrm{H}, \mathrm{NH}) ;{ }^{13} \mathrm{C}$ 
NMR $\left(\mathrm{CDCl}_{3}, 50 \mathrm{MHz}\right) \delta 9.00(\mathrm{C}-7), 12.25\left(\mathrm{CH}_{3}\right), 17.59$ (C-4), 62.84 (C-5), 63.04 (C-3), 72.95 and $73.01\left(\mathrm{CH}_{2}\right), 73.74(\mathrm{C}-2), 74.23\left(\mathrm{CH}_{2}\right), 76.78(\mathrm{C}-1), 109.25(\mathrm{C}-5$ ') , 127.60, 127.77, 128.20, 128.35 and 128.45 (aromatic $\mathrm{CH}$ ), 136.80 and 137.90 (aromatic C), 138.26 (C-6'), 150.12 (C2'), 163.52 (C-4'); Anal. Calc. for $\mathrm{C}_{26} \mathrm{H}_{28} \mathrm{~N}_{2} \mathrm{O}_{6}$ : C, 67.23; H, 6.08\%. Found: C, 67.36; H, 6.04.

1-\{(1R,3R,4R,5S,6R)-4,5-Dihydroxy-1-(hydroxymethyl)-2-oxa-bicyclo[4.1.0]heptan-3yl\}pyrimidine-2,4(1H,3H)-dione (9). A solution of $8(210 \mathrm{mg}, 0.53 \mathrm{mmol})$ in methanolic ammonia $(12 \mathrm{ml})$ was stirred at room overnight, then concentrated under reduce pressure and purified by flash chromatography to give 5 as a yellow syrup $\left(78 \%\right.$ yield): ${ }^{1} \mathrm{H} \mathrm{NMR}\left(\mathrm{CD}_{3} \mathrm{CN}\right.$, $500 \mathrm{MHz}) \delta 0.77(\mathrm{dd}, 1 \mathrm{H}, J=6.0,10.5 \mathrm{~Hz}, \mathrm{H}-7 \mathrm{a}), 0.91(\mathrm{dd}, 1 \mathrm{H}, J=6.5,7.0 \mathrm{~Hz}, \mathrm{H}-7 \mathrm{~b}), 1.52$ (ddd, $1 \mathrm{H}, J=6.5,7.0,10.5 \mathrm{~Hz}, \mathrm{H}-4), 3.28-3.70$ (AB system, 2H, $J_{\mathrm{AB}}=12.0 \mathrm{~Hz}, \mathrm{H}-6 \mathrm{a}, \mathrm{H}-6 \mathrm{~b}$ ), $3.38(\mathrm{dd}, 1 \mathrm{H}, J=8.0,10.0 \mathrm{~Hz}, \mathrm{H}-2), 4.17(\mathrm{dd}, 1 \mathrm{H}, J=7.0,8.0 \mathrm{~Hz}, \mathrm{H}-3), 5.44$ (d, $1 \mathrm{H}, J=10.0$ $\mathrm{Hz}, \mathrm{H}-1), 5.63$ (d, 1H, $J=8.2 \mathrm{~Hz}, \mathrm{H}-5$ ') 7.41 (d, 1H, $J=9.2 \mathrm{~Hz}, \mathrm{H}-6$ '); ${ }^{13} \mathrm{C}$ NMR $\left(\mathrm{CD}_{3} \mathrm{CN}, 50\right.$ MHz) $\delta 15.26$ (C-4), 24.00 (C-7), 66.90 (C-5), 72.18 (C-6), 72.24 (C-3), 72.73 (C-2), 83.02 (C1), 103.06 (C-5'), 141.72 (C-6'), 158.43 (C-2'), 161.22 (C-4'); Anal. Calc. for $\mathrm{C}_{11} \mathrm{H}_{14} \mathrm{~N}_{2} \mathrm{O}_{6}$ : C, 58.89; H, 5.22\%. Found: C, 58.83; H, 5.19.

General procedure for the debenzylation of cyclopropanated nucleosides 10a,b and 11a,b. To a solution of cyclopropanated nucleosides 10a,b and 11a,b $(0.90 \mathrm{mmol})$ in ethanol $(3 \mathrm{~mL})$ was added a spatula tip of $\mathrm{Pd} / \mathrm{C}$ and the suspension was stirred under $\mathrm{H}_{2}$ atmosphere for $12 \mathrm{~h}$. After this time the suspension was filtered and the filtrate was evaporated at reduced pressure to give, with nearly quantitative yields, the corresponding deprotected derivatives $\mathbf{9}, \mathbf{1 2 b}$ and 13a,b.

1-\{(1R,3R,4R,5S,6R)-4,5-Dihydroxy-1-(hydroxymethyl)-2-oxa-bicyclo[4.1.0]heptan-3-yl\}-5methylpyrimidine-2,4(1H,3H)-dione (12b). Amorphous solid, yield 90\%, $[\alpha]_{\mathrm{D}}^{25}+69.22$ (c 0.3 , $\mathrm{MeOH}) .{ }^{1} \mathrm{H}$ NMR $\left(\mathrm{CD}_{3} \mathrm{OD}, 500 \mathrm{MHz}\right) \delta 0.84(\mathrm{dd}, 1 \mathrm{H}, J=6.0,10.0 \mathrm{~Hz}, \mathrm{H}-7 \mathrm{a}), 1.02(\mathrm{t}, 1 \mathrm{H}, J=$ $7.0 \mathrm{~Hz}, \mathrm{H}-7 \mathrm{~b}$ ), 1.58 (ddd, 1H, $J=6.5,7.0,10.0 \mathrm{~Hz}, \mathrm{H}-4), 1.87$ (d, $J=1.0 \mathrm{~Hz}, \mathrm{CH}_{3}$ ), 3.39-3.75 (AB system, 2H, $\left.J_{\mathrm{AB}}=12.5 \mathrm{~Hz}, \mathrm{H}-6 \mathrm{a}, \mathrm{H}-6 \mathrm{~b}\right), 3.47$ (dd, $\left.1 \mathrm{H}, J=8.0,9.5 \mathrm{~Hz}, \mathrm{H}-2\right), 4.21$ (dd, $1 \mathrm{H}, J$ $=7.0,8.0 \mathrm{~Hz}, \mathrm{H}-3), 5.52(\mathrm{~d}, 1 \mathrm{H}, J=9.5 \mathrm{~Hz}, \mathrm{H}-1), 7.41\left(\mathrm{~d}, 1 \mathrm{H}, J=1.0 \mathrm{~Hz}, \mathrm{H}-6\right.$ '); ${ }^{13} \mathrm{C}$ NMR $\left(\mathrm{CD}_{3} \mathrm{OD}, 50 \mathrm{MHz}\right) \delta 12.37\left(\mathrm{CH}_{3}\right), 15.47(\mathrm{C}-7), 23.60(\mathrm{C}-4), 66.74(\mathrm{C}-6), 53.15(\mathrm{C}-5), 71.72(\mathrm{C}-$ 3), 76.78 (C-2), 83.08 (C-1), 112.85 (C-5'), 135,11 (C-6’), 157.02 (C-2'), 162.21 (C-4'); Anal. Calc. for $\mathrm{C}_{12} \mathrm{H}_{16} \mathrm{~N}_{2} \mathrm{O}_{6}$ : C, 50.70; $\mathrm{H}, 5.67 \%$. Found: C, 50.77; H, 5.62.

1-\{(1R,3S,4R,5S,6R)-4,5-Dihydroxy-1-(hydroxymethyl)-2-oxa-bicyclo[4.1.0]heptan-3yl\}pyrimidine-2,4(1H,3H)-dione (13a). Amorphous solid, yield $89 \%,[\alpha]_{\mathrm{D}}^{25}-38.75$ (c 0.08 , $\mathrm{MeOH}) .{ }^{1} \mathrm{H}$ NMR $\left(\mathrm{CD}_{3} \mathrm{OD}, 500 \mathrm{MHz}\right) \delta 0.80(\mathrm{dd}, 1 \mathrm{H}, J=6.0,10.0 \mathrm{~Hz}, \mathrm{H}-7 \mathrm{a}), 1.02(\mathrm{dd}, 1 \mathrm{H}, J=$ 6.0, 7.0 Hz, H-7b), 1.48 (ddd, $1 \mathrm{H}, J=6.5,7.0,10.0 \mathrm{~Hz}, \mathrm{H}-4), 3.48-3.80$ (AB system, $2 \mathrm{H}, J_{\mathrm{AB}}=$ $11.5 \mathrm{~Hz}, \mathrm{H}-6 \mathrm{a}, \mathrm{H}-6 \mathrm{~b}$ ), 3.35 (dd, 1H, $J=2.0,4.5 \mathrm{~Hz}, \mathrm{H}-2), 4.13$ (dd, 1H, $J=4.5,7.5 \mathrm{~Hz}, \mathrm{H}-3$ ), 5.48 (d, $1 \mathrm{H}, J=2.0 \mathrm{~Hz}, \mathrm{H}-1), 5.59$ (d, $1 \mathrm{H}, J=8.2 \mathrm{~Hz}, \mathrm{H}-5$ '), 7.89 (d, 1H, $J=1.5 \mathrm{~Hz}, \mathrm{H}-6$ '); ${ }^{13} \mathrm{C}$ NMR (CD $3 \mathrm{OD}, 50 \mathrm{MHz}) \delta 15.31(\mathrm{C}-4), 23.75$ (C-7), 53.27 (C-5), 66.84 (C-6), 71.62 (C-3), 78.28 (C-2), 82.58 (C-1), 111.85 (C-5’), 134,61 (C-6’), 155.12 (C-2'), 161.11 (C-4'); Anal. Calc. for $\mathrm{C}_{11} \mathrm{H}_{14} \mathrm{~N}_{2} \mathrm{O}_{6}$ : C, 48.89; H, 5.22\%. Found: C, 48.95; H, 5.26. 
1-\{(1R,3S,4R,5S,6R)-4,5-Dihydroxy-1-(hydroxymethyl)-2-oxa-bicyclo[4.1.0]heptan-3-yl\}-5methylpyrimidine-2,4(1H,3H)-dione (13b). Amorphous solid, yield 90\%, $[\alpha]_{\mathrm{D}}^{25}-131.54(\mathrm{c} 0.2$, $\mathrm{MeOH}) .{ }^{1} \mathrm{H}$ NMR $\left(\mathrm{CD}_{3} \mathrm{OD}, 500 \mathrm{MHz}\right) \delta 0.76(\mathrm{dd}, 1 \mathrm{H}, J=6.5,10.0 \mathrm{~Hz}, \mathrm{H}-7 \mathrm{a}), 0.97(\mathrm{t}, 1 \mathrm{H}, J=$ $6.5 \mathrm{~Hz}, \mathrm{H}-7 \mathrm{~b}$ ), 1.38 (ddd, $1 \mathrm{H}, J=6.5,7.0,10.0 \mathrm{~Hz}, \mathrm{H}-4), 1.88$ (d, $J=1.5 \mathrm{~Hz}, \mathrm{CH}_{3}$ ), 3.51-3.85 (AB system, $\left.2 \mathrm{H}, J_{\mathrm{AB}}=12.0 \mathrm{~Hz}, \mathrm{H}-6 \mathrm{a}, \mathrm{H}-6 \mathrm{~b}\right), 3.47$ (dd, $1 \mathrm{H}, J=1.5,7.5 \mathrm{~Hz}, \mathrm{H}-2$ ), 4.33 (dd, $1 \mathrm{H}, J$ $=7.0,7.5 \mathrm{~Hz}, \mathrm{H}-3), 5.78(\mathrm{~d}, 1 \mathrm{H}, J=1.5 \mathrm{~Hz}, \mathrm{H}-1), 7.89$ (d, $1 \mathrm{H}, J=1.5 \mathrm{~Hz}, \mathrm{H}-6$ '); ${ }^{13} \mathrm{C}$ NMR $\left(\mathrm{CD}_{3} \mathrm{OD}, 50 \mathrm{MHz}\right) \delta 12.37\left(\mathrm{CH}_{3}\right), 15.47(\mathrm{C}-7), 23.60(\mathrm{C}-4), 66.74(\mathrm{C}-6), 53.15(\mathrm{C}-5), 71.72(\mathrm{C}-$ 3), 76.78 (C-2), 83.08 (C-1), 112.85 (C-5'), 135,11 (C-6’), 157.02 (C-2'), 162.21 (C-4'); Anal. Calc. for $\mathrm{C}_{12} \mathrm{H}_{16} \mathrm{~N}_{2} \mathrm{O}_{6}$ : C, 50.70; H, 5.67\%. Found: C, 50.77; H, 5.62.

\section{Antiviral and cytotoxicity assay for HSV}

The newly synthesized nucleosides were evaluated for their activity against HSV-1 and HSV-2 by plaque reduction assay in VERO cells using a methodology reported in the literature. ${ }^{13}$ Cytotoxicity assays were conducted in rapidly dividing Vero cells, as reported. ${ }^{13}$

\section{Evaluation of toxicity and apoptosis}

Toxicity was evaluated by a standard viability assay, using the trypan blue exclusion test. Normally, apoptosis was evaluated by morphological analysis of the cells, performed following staining with acridine orange as previously described. ${ }^{14}$ Briefly, over 600 cells, including those showing typical apoptotic characteristics, were counted using a fluorescence microscope. The identification of apoptotic cells was based on the presence of uniformly stained nuclei showing chromatin condensation and nuclear fragmentation. In some experiments, apoptosis was detected by flow cytometric analysis of isolated nuclei, following staining with propidium iodide, on a Becton Dickinson FAC Scan Analytic Flow Cytometer, as previously described. ${ }^{15}$

\section{Acknowledgments}

We thank the M.I.U.R. and C.N.R. for their partial financial support.

\section{References}

1. Verheggen, I.; Van Aerschot, A.; Toppet, S.; Snoeck, R.; Janssen, G.; Balzarini, J.; De Clercq, E.; Herdewijn, P. J. Med. Chem. 1993, 36, 2033.

2. Verheggen, I.; Van Aerschot, A.; Van Meervelt, L.; Rozenski, J.; Wiebe, L.; Snoeck, R.; Andrei, G.; Balzarini, J.; Claes, P.; De Clercq, E.; Herdewijn, P. J. Med. Chem. 1995, 38, 826. 
3. Ostrowski, T.; Wroblowski, B.; Busson, R.; Rozenski, J.; De Clercq, E.; Bennet, M. S.; Champness, J. N.; Summers, W. C.; Sanderson, M. R.; Herdewijn, P. J. Med. Chem. 1998, $41,4343$.

4. Haouz, A.; Vanheusden, V.; Munier-Lehmann, H.; Froeyen, M.; Herdewijn, P.; Van Calenbergh, S.; Delarue, M. J. Biol. Chem. 2003, 278, 4963.

5. Haouz, A.; Vanheusden, V.; Munier-Lehmann, H.; Froyen, M.; Herdewijn, P.; Van Calenbergh, S.; Delarue, M. J. Biol. Chem. 2003, 278, 4963.

6. Vastmans, K.; Pocket, S.; Peyes, A.; Kerremans, L.; Van Aerschot, A.; Hendrix, C.; Merliere, P.; Herdewijn, P. Biochemistry 2000, 39, 12757.

7. Jaggannadh, B.; Reddy, D. V.; Kunwar, A. C. J. BioChem. Biophys. Res. Commun. 1991, 179, 386.

8. Marquez, V. E.; Ezzitouni, A; Russ, P.; Siddiqui, M. A.; Ford, H. J.; Feldman, R. J.; Mitsuya, H.; George, C.; Barchi, J. J., Jr. J. Am. Chem. Soc. 1998, 120, 2780.

9. Catelani, G.; Corsaro, A.; D’Andrea, F.; Fisichella, S.; Mariani, M.; Pistarà, V. Environ. Sci. \& Pollut. Res. 2003, 10, 325.

10. Corsaro, A.; Chiacchio, U.; Adamo, R.; Pistarà, V.; Rescifina, A.; Romeo, R.; Catelani, G.; D’Andrea, F.; Mariani, M.; Attolino, E., Tetrahedron, 2004, 60, 3787.

11. (a) Hoberg, J. O.; Bozell, J. J. Tetrahedron Lett. 1995, 36, 6831. (b) Simmons, H. E.; Cairns, T. L.; Vladuchik, S. A.; Hoiness, C. M. Org. Reactions 1973, $20,1$.

12. .Vorbrüggen, H.; Krolikiewicz, K.; Bennua, B. Chem. Ber. 1981, 114, 1234

13. Schinazi, R. F.; Peters, J.; Williams, C. C.; Chance, D.; Nahmias, A. J. Antimicrob. Agents Chemother. 1982, 22, 499.

14. Mastino, A.; Sciortino, M. T.; Medici, M. A.; Perri, D.; Ammendolia, M. G.; Grelli, S.; Amici, C.; Pernice, A. Cell. Death. Differ. 1997, 4, 649.

15. Matteucci, C.; Grelli, S.; De Smaele, E.; Fontana, C.; Mastino, A. Cytometry, 1999, 35, 145. 